\title{
RORTY : ¿CABE HABLAR DE LA INDEPENDENCIA CAUSAL DEL MUNDO DESDE UNA ONTOLOGÍA PANRELACIONISTA? 1
}

\author{
RORTY: ¿IS IT POSSIBLE TO FIT THE CAUSAL INDEPENDENCE OF THE \\ WORLD INTO A PANRELATIONIST ONTOLOGICAL FRAMEWORK?
}

\author{
Óscar L. González-Castán
}

Recibido: $15 / 04 / 2015$

Aceptado: 09/07/2015

\section{RESUMEN}

Richard Rorty ha reflexionado sobre tres tesis fundamentales para su programa filosófico que él ve, además, profundamente relacionadas: (1) la existencia de un mundo causalmente independiente de nuestras creencias y deseos, (2) el papel que juega el mundo y los sistemas de creencias en la formación de nuestras plurales descripciones del mundo y (3) la idea, ligada con el panrelacionismo, de que el mundo no tiene ninguna esencia. En este trabajo argumentaré que, sin embargo, el conjunto de sus argumentaciones plantea dificultades difícilmente superables.

PALABRAS CLAVE: realismo, causalidad, creencia, anti-esencialismo, panrelacionismo.

\section{ABSTRACT}

\footnotetext{
${ }^{1}$ Este artículo se inscribe dentro del proyecto de investigación del Ministerio de Ciencia e Innovación titulado "Normatividad y praxis. El debate actual después de Wittgenstein" (FFI 2010-15975). También es una contribución al Programa de Creación y Consolidación de Grupos de Investigación UCM-Santander y, en concreto, al proyecto "Razonabilidad versus racionalidad en el marco de una propuesta de reunificación de la cultura" (GR 3/14).

Doy las gracias a los evaluadores anónimos de la revista por sus sugerencias y comentarios que me han servido para intentar mejorar el contenido de este artículo.
} 
Richard Rorty has reflected about three theses that are central for his philosophical agenda and he considers as profoundly intertwined: (i) The existence of a world that is causally independent of our beliefs and desires, (ii) the role that both the world and the belief systems play in the formation of our plural descriptions of the world, and (iii) the idea, related with his panrelationism, that the world does not have any essence whatsoever. However, in this paper I shall argue that Rorty`s argumentations do not provide a coherent set of ideas.

KEYWORDS: realism, causality, belief, anti-essentialism, panrelationism.

\section{INTRODUCCIÓN}

El pensamiento de Richard Rorty aborda muchos de los grandes problemas de la filosofía con una resolución y amplitud de miras dignas de ser envidiadas por muchos filósofos contemporáneos, más limitados y locales en los problemas que tratan y en la forma de pensarlos. Rorty, sin embargo, no tenía un sistema pero sí un programa, un horizonte que bañaba su talante filosófico. Hacia ese lugar quiero dirigirme para mostrar que este filósofo reunió alrededor de alguna de sus tesis fundamentales ciertas afirmaciones que, en sus conexiones mutuas, plantean más problemas de los que él pensaba. Argumentaré concretamente que hay una tensión de fondo no resuelta entre la tesis de la independencia causal del mundo y el hecho de que existe en toda descripción una mezcolanza entre el mundo y las creencias de los sujetos que forman parte de una comunidad etnocéntrica. Si, de acuerdo con la argumentación de Rorty, se busca apoyo a la independencia causal del mundo y se exploran sus consecuencias, entonces sufre la tesis de la mezcolanza. Si, por el contrario y en sentido opuesto, se busca reforzar la tesis de la mezcolanza y sus derivaciones, especialmente las que tienen que ver con el antiesencialismo y el panrelacionismo, se debilita entonces problemáticamente la tesis de la independencia causal del mundo. El resultado es la inquietud filosófica que produce un conjunto de ideas altamente atractivo pero no bien atado del que Rorty parece que nunca, irónicamente, llegó a desconfiar después de su giro hermenéutico en tanto que expresión de su vocabulario fundamental. ${ }^{2}$

${ }^{2}$ Este tipo de crítica general no es nuevo cuando se habla de Rorty. Por ejemplo, Alasdair MacIntyre, entre otros muchos, comentaba ya que el estilo retórico de Rorty hace que "se tengan que rellenar vacíos argumentativos, que haya lugares en los que todo depende de los detalles, pero en los que estos detalles están ausentes, y que haya incoherencias que tendrán que resolverse" (MACINTYRE 1990, p. 710). 


\section{REALISMO, CAUSALIDAD Y MEZCOLANZA}

Rorty sostiene algunas tesis de sabor claramente realista. La fundamental es que el pragmatista post-kuhniano es coherentista pero no idealista porque cree, "tanto como el realista, que hay objetos causalmente independientes de las creencias y deseos humanos" (RORTY 1991c, p. 101). El de Rorty es un robusto realismo de sentido común, el realismo característico de la actitud natural en sentido husserliano. Este realismo le sirve a Rorty, primero, para apartarse de un plumazo de su opuesto idealista y, segundo, para no negar en todo sentido posible que hay algo que se nos impone impertinentemente en forma de resistencias, facilidades y dificultades, una donación originaria de algo real distinto del ser humano y de su sistema de creencias. Hay mundo, naturaleza, y no simple fantasmagoría fenoménica. Cargado de razón, Habermas decía que "el giro pragmático no da cabida a la duda respecto de la existencia de un mundo independiente de nuestras descripciones. Antes bien, de Peirce a Wittgenstein, la ociosa duda cartesiana ha sido rechazada como una contradicción performativa" (HABERMAS, p. 39).

Protegido por el rebufo de James, Dewey y Davidson, Rorty simplemente añadiría, para evitar malentendidos respecto de su postura realista general, que es imposible realizar un análisis factorial de cualquiera de las variadísimas descripciones humanas del mundo con el fin de establecer con nitidez cuánto en ellas se debe al mundo y cuánto a las creencias de partida del grupo humano que las elabora y propone. Si se lograra este objetivo se habría conseguido obtener una clase reducida de descripciones en las que el ser humano no ha puesto nada de su cosecha, en la que se ha convertido en un puro espejo neutral del mundo, en una forma sin forma, como decía Aristóteles para

La amplísima literatura especializada que existe sobre Rorty ha tratado los problemas que abordo en este trabajo - en realidad no queda seguramente nada en la obra de Rorty que no se haya escrutado de alguna manera--- pero no he encontrado un tratamiento pormenorizado, explícito y concentrado de las relaciones problemáticas que establezco entre los temas que desplegaré. Así, por ejemplo, en la literatura en español más reciente José Antonio GarcíaLorente, desde claves clásicas griegas y medievales actualizadas, trata críticamente el panrelacionismo pero no lo pone en relación con el problema del descriptivismo y de la independencia causal del mundo (GARCÍA LORENTE 2012, p. 180-186). Cfr. (GARCíA LORENTE 2011). En otros estudios recientes en español sobre la obra de Rorty pasa también lo mismo. Cfr. (Del CASTILlO y LÓPEZ 2014), (COLOMINA y RAGA 2010) y (KALPOKAS 2005).

La misma situación puede encontrarse en la literatura anglosajona. Como muestra puede consultarse la monumental y, para Rorty, postuma obra de (AUXIER y HAHN 2010) en donde el panrelacionismo ni siquiera aparece en el índice de nombres y conceptos. 
caracterizar el alma humana y permitir, así, su posibilidad de conocimiento objetivo del mundo.

La imposibilidad de hacer este análisis factorial se debe a que no podemos separar la contribución causal que hace el mundo al proceso que desencadena o rinde una determinada descripción lingüística respecto de nuestra propia contribución a este proceso, la cual es, en parte, natural, pues depende del tipo de organismo que somos y, en parte, histórico-lingüística, pues procede también de la tradición cultural a la que pertenecemos (RORTY 1995, p. 295). ${ }^{3}$ Sólo la unidad atemporal del método y su aplicación trasversal a toda actividad humana a la que se ha llamado "ciencia" puede llevarnos a acuerdos, si no unánimes, sí significativamente amplios, y a sincronizar el contenido de todos los sistemas de creencias porque, gracias a tal método, no quedaría en ellos nada de la posible escoria cognoscitiva que las distintas tradiciones y familias humanas han añadido a nuestros intentos de descripción neutral del mundo. Gracias a dicho método habríamos logrado el análisis factorial que se buscaba. Dado que para Rorty no hay un método general de investigación científica que no esté cargado históricamente con los procedimientos de justificación de un ethnos particular, ni métodos parciales que no sean con frecuencia revisables, hay que concluir una vez más que nuestros juicios sobre la realidad sólo pueden ser interpretativos. En ellos siempre hay una mezcolanza insuperable entre el mundo y las creencias.

Así planteada, la tesis de la mezcolanza entre el mundo y nuestro sistema de creencias puede ser pensada como una tesis meramente epistemológica que afectaría solamente a nuestra capacidad para conocer este mundo que es independiente de nuestros sistemas de creencias, los cuales, para gran parte de la tradición filosófica de raigambre ilustrada, son casi siempre distorsionadores.

\footnotetext{
${ }^{3}$ La tesis citada es de raigambre fuertemente hegeliana. En la Introducción a la Fenomenología del Espíritu Hegel critica la posibilidad de sustraer en nuestros juicios cognitivos lo que aporta el medio o instrumento del conocimiento, potencialmente distorsionador del objeto, a lo que pone el objeto de conocimiento. Conocer auténticamente un objeto sería el resultado de esa labor de resta. Sin embargo, Hegel sostiene que representarse de esta manera el conocimiento es absurdo.

En palabras de Rorty y como parte de su intento de aclarar sus acuerdos y desacuerdos con Putnam, quien le había acusado de relativista, "los elementos de lo que llamamos 'lenguaje' o 'mente' penetran tan profundamente en lo que llamamos 'realidad' que el mismo proyecto de representarnos a nosotros mismos como 'cartógrafos de algo que es independiente de la mente’ está fatalmente comprometido desde el principio” (RORTY 1993, p. 443).
} 
Esta lectura epistemológica tendría, además, una doble vertiente. La primera declararía, como ya sabemos, que no podemos saber cuánto en una descripción se debe al componente "mundo" y cuánto al componente "sistema de creencias". Como consecuencia de esta primera faceta, se afirmaría, en segundo lugar, que no se puede conocer el mundo tal y como es en sí, bien porque no podemos conocerlo en absoluto -estaríamos en una versión del escepticismo radical--, bien porque no podemos conocerlo en-sí de una forma independiente de nuestros intereses, valores y tradiciones, es decir, de nuestros juegos de lenguaje $\mathrm{y}$, por tanto, de la intermediación distorsionadora inevitable de nuestros marcos de pensamiento lingüísticos y culturales. En este último caso estaríamos dentro de una versión del escepticismo agnóstico a la que nos conduciría la tesis de la mezcolanza creencias/mundo fruto de una mediación lingüístico/cultural entre el mundo-en-sí y cada uno de nosotros. Rorty, sin embargo, rechaza cualquiera de estas dos formas de escepticismo a las que conduce la interpretación epistemológica de su filosofía. Cualquiera de los dos casos haría del mundo un mundus absconditus, un mundo constante y constituido desde siempre en su versión única y definitiva, según la famosa metáfora de James, pero que se retraería pertinazmente de nuestros intentos de conocimiento, como le dijo McDowell a Rorty que pasaría con su filosofía pragmatista (MCDOWELL, p. 110). Sin embargo, Rorty introduce otras consideraciones que impiden quedarse limitados a esta lectura meramente epistemológica, ligada al escepticismo, para tener que abrirnos también a una lectura ontológica complementaria que está a su base. En virtud de esta ampliación Rorty pretenderá reafirmar la primera mitad de la interpretación epistemológica sin tener que aceptar necesariamente la segunda. Se trataría, por tanto, de seguir sosteniendo la tesis de la imbricación creencias/mundo y sus implicaciones sin recalar forzosamente en alguna variación del escepticismo. Los pasos para realizar esta operación son, según creo, los siguientes.

Rorty se desmarca rotundamente de una forma de entender el realismo de partida que podría parecer su consecuencia inevitable o su acompañante fiel. En ninguna actividad humana, ni siquiera en la indagación científica, se puede pretender "hallar la naturaleza de algo que esté fuera de la trama de creencias y deseos" (RORTY 1991c, p. 96). Esta nueva tesis es, en parte, el reverso necesario de la idea de que el fin de la investigación no puede ser la verdad, la descripción aséptica, adecuada y objetiva de la realidad. Sin embargo, con ella se añade algo más, afirmado por Rorty en muchísimas otras ocasiones, que tiene un cariz marcadamente ontológico, a saber, que, de hecho, el mundo no 
tiene ningún en-sí cuya naturaleza algunas personas desean conocer paulatinamente pues solamente con su satisfacción se cumplirían las aspiraciones cognoscitivas humanas más profundas. El antiesencialismo de Rorty consiste en decir que las "cosas" no tienen ninguna esencia o naturaleza y que, por lo tanto, no hay nada inalterable que conocer. No hay ningún mundo-en-sí frente al cual tengamos la labor o el deseo epistémico de conocerlo con verdad en una representación ajustada, de elaborar para él un ropaje de ideas que se le ciña perfectamente (ein wohlpassendes Ideenkleid), como ya decía críticamente Husserl respecto de ciertas autoimágenes extendidas de la ciencia natural (HuSSERL 1954, p. 51). Es por este motivo por el que no tiene sentido --y no simplemente que sea imposible para nosotros por diversos motivos escépticos-- ponerse a buscar, con la ayuda de un método, el conjunto de las descripciones neutrales del mundo en las que no estaría implicado ningún factor humano. Para Rorty, sólo cuando se mantiene el supuesto ontológico de la existencia de un mundo en-sí es cuando aparecen inevitablemente los enojosos problemas filosóficos con el escepticismo que podrían derivarse de su tesis de la mezcolanza mundo/creencias.

\section{PANRELACIONISMO ANTIESENCIALISTA, PLURALISMO CONCEPTUAL Y CONTEXTUALISMO}

Para comprender mejor la relación entre el antiesencialismo y las descripciones humanas de un mundo que no tiene ningún en-sí pero en las que se amalgaman las tradiciones histórico-lingüísticas particulares con un mundo causalmente independiente es importante atender con más detalle tanto al panrelacionismo de Rorty como a su conexión con el pluralismo conceptual. Fundamentalmente Rorty desemboca en la tesis de que el mundo no tiene ningún en-sí por dos vías diferenciadas que él ve enteramente emparentadas pero que, sin embargo, tienen alcances muy distintos y justificaciones muy variadas aunque, en conjunto, tienen consecuencias perniciosas para entender bien la independencia causal del mundo.

\subsection{ANTIESENCIALISMO Y PANRELACIONISMO}

La afirmación de que el mundo no tiene ningún en-sí absoluto es el núcleo del antiesencialismo el cual está fuertemente ligado en Rorty con el 
panrelacionismo como tesis ontológica de fondo de su filosofía. Rorty formula con fuerza y concisamente de esta manera su panrelacionismo ontológico el cual sería válido para cualquier tipo de objeto que se presente a nuestra consideración:

[El panrelacionismo es] "pensar que las cosas son como son en virtud de las relaciones que mantienen con las demás cosas [...] [Es tratar] de sustituir las distintas imágenes del mundo construidas con la ayuda de las oposiciones griegas [necesario/contingente, realidad/apariencia] por la imagen de un flujo de relaciones en cambio constante, relaciones cuyos términos son, a su vez, también disolubles en los nexos de otras nuevas relaciones" (RORTY 2000a, p. 140).

Por tanto, el panrelacionismo que Rorty parece tener en mente se da estrechamente la mano con el antiesencialismo. Para él el panrelacionismo es antiesencialista de tal forma que la mejor manera de defender el antiesencialismo es, a su vez, mediante alguna versión del panrelacionismo. Las relaciones son previas, por decirlo así, a los objetos los cuales, ahora, son simplemente los nudos que ellas forman en sus interconexiones mutuas y no aquello que preexiste y puede entrar posteriormente en relación con otros objetos. Por este motivo las relaciones constituyen a los objetos y no al revés. ${ }^{4}$

\footnotetext{
${ }^{4}$ Hay que tener en cuenta que en la discusión ontológica y metafísica actual hay formas esencialistas del panrelacionismo que Rorty no considera polémicamente en la formulación de sus tesis. Son familiares las teorías denominadas "estructuralistas" en filosofía de las matemáticas y en filosofía de la física que han sostenido que todo lo que hay -en los respectivos dominios- son relaciones, de manera que lo que denominamos como "objetos" son simplemente nodos en las estructuras resultantes por la complexión de relaciones. Esto, por sí mismo, sin embargo, no tiene ninguna implicación respecto a cuál sea la esencia de los "objetos", comoquiera que estos "objetos" sean finalmente concebidos, ni mucho menos para la esencia de la estructura completa. Supóngase que hay ciertas relaciones "básicas" que especifican la estructura fundamental. Los nodos que resulten en tal estructura contarán como "objetos". Cada una de las relaciones integrantes en esta estructura fundamental resultarán, por lo tanto, esenciales a los "objetos", pues los constituyen. Esto no sería óbice para que, una vez constituidos los "objetos" como nodos en la estructura fundamental, existan relaciones entre tales nodos pero esta vez de carácter contingente. Por supuesto, en una ontología específica esto debería ser precisado y debería ofrecerse una motivación para cada una de estas postulaciones. La cuestión es que se trataría de una ontología en la que la única categoría postulada sería la de relación, pero esto no impediría que hubiese propiedades (poliádicas en este caso) esenciales y accidentales. En cualquier caso, dado el fin de este ensayo, dejaré al
} 
En el texto citado Rorty sostiene, además, que si proponemos otras relaciones nuevas y pensamos los objetos en función de ellas, cambiaremos esos mismos objetos o, incluso, constituiremos otros completamente novedosos aunque puedan llevar el mismo nombre que los anteriores.

Rorty da dos ejemplos fundamentales de esta atmósfera panrelacionista en la que viven los objetos, uno con el que discrepo y otro con el que simpatizo. Según el primer ejemplo, más bien una analogía cuyo alcance es limitado y fallido, debemos pensar todo objeto como pensamos matemáticamente en el número 17. Siempre pensamos en este número, como en cualquier otro número natural, en función de muy variadas relaciones y, por lo tanto, descripciones: el número que sigue al 16 y antecede al 18 -la relación más habitual con la que lo pensamos--, la suma de 6 y 11, la raíz cuadrada de 289, el cuadrado de 4,123105, etc. Ante esta situación Rorty concluye, como debemos concluir para todo otro "objeto" del tipo que sea, que ninguna de estas descripciones ofrece

"una pista de la intrínseca diecisieticidad del diecisiete, la característica única que hace que sea el número que justamente es. Por cuál de esas descripciones optamos es, obviamente, un asunto sobre qué propósito tenemos en mente, la situación particular responsable de que pensáramos en el 17 en primer lugar" (RORTY 2000a, p. 144).

Sin embargo, este ejemplo no es del todo bueno porque no es cierto, como declara la tesis del panrelacionismo, que si variamos las relaciones en que está subsumido el número 17 y que desembocan, como en un cruce de carreteras, siempre en él, estemos pensando en el número 17 aunque de otra manera. Si dices que el 17 es el número que sigue al 18 y está antes del 20, habrás cambiado todo lo que quieras las relaciones pero no estarás pensando en el 17, aunque lo llames así. Por decirlo resumidamente, la relación "seguir al 18 pero estar antes del 20" no desemboca en el número 17.

Precisamente es esto lo que no pasa con el otro ejemplo con el que, como he comentado, sí simpatizo. Pensar en qué es "la mujer" o cualquier otra realidad social no debe consistir en pensar en una esencia natural y/o social, sino en pensar que si cambiamos las relaciones sociales en las que participan y

margen el tratamiento de las tesis de Rorty en el contexto de la discusión ontológica sistemática actual, pues esto llevaría a plantear un trabajo totalmente distinto. 
pueden participar con sus acciones las mujeres --fundamentalmente porque un grupo cada vez más amplio de ellas ha luchado tenaz, inteligentemente y con sufrimiento para hacerlo--, entonces cambiará nuestra concepción de las mujeres como ser biológico y social. En lugar de pensar monotemáticamente de forma patriarcal que la mujer es por esencia esposa, ama de casa, madre y cuidadora, es pensarla como alguien capaz de, y con derecho a, formar parte de otras muchas relaciones sociales como votante, catedrática de universidad, diputada, alto directivo, multimillonaria, filósofa, con derecho al mismo salario por el mismo trabajo, torturadora (como en la cárcel de Abu Ghraib), etc. Aquí, "ser mujer" se diluye de formas muy plurales en las muchas relaciones sociales en las que ellas pueden participar y que pueden elegir dentro de límites inevitables como pasa en toda elección. Se trata, por tanto, de expandir de formas novedosas "el espacio lógico" de las relaciones en las que intervienen activamente las mujeres gracias a la creación de nuevos roles sociales, con sus correspondientes formas de vida y los vocabularios aptos para describirlas (RORTY 1998b, p. 204).

Estas diferencias entre los objetos "el número 17" y "las mujeres" son indicativas de la necesidad de preguntarse críticamente si todos los objetos son susceptibles de ser pensados resumidamente bajo la tesis ontológica panrelacionista antiesencialista, en su versión rortyana. Parece que las cosas en este ámbito ontológico son más complejas y que hace falta introducir muchas más distinciones y matizaciones como, por ejemplo, la distinción entre objetos matemáticos, objetos físicos, objetos cotidianos y objetos sociales, por mucho que Rorty haya tratado siempre de difuminar todo lo posible este tipo de distinciones al criticar que haya cosas que son independientes del contexto y cosas que sí dependen del contexto (RORTY 1991b). Lo único que habría mostrado Rorty es que el panrelacionismo está fuertemente relacionado con el pluralismo descriptivo -el "17" y la "mujer" se pueden describir de múltiples maneras, más o menos complejas, con resultados diversos-pero no habría mostrado que todo objeto es susceptible de ser pensado de formas novedosas, alterando su contenido, es decir, su lugar en una red de relaciones, en función de otras nuevas relaciones. Con esta reflexión simplemente estoy queriendo indicar que estamos ante un dilema. Si proponemos el número 17 como modelo paradigmático para pensar en el panrelacionismo y extender su alcance a todo otro tipo de objeto, entonces el modelo mismo tiene problemas y, por consiguiente, se hace problemática su generalización pues no todos los objetos parecen comportarse exactamente como su patrón. Si, por el contrario, 
proponemos a "la mujer" como modelo del panrelacionismo, entonces no queda claramente especificado en Rorty que este modelo alternativo sirva para todo otro tipo de objeto. Quedaría por resolver, por ejemplo, el estatuto ontológico de objetos matemáticos como el 17. Una salida razonable posible a esta situación sería decir que el mundo con el que Rorty pretende lidiar y respecto del cual pretende hacer valer en primer lugar su panrelacionismo es el mundo causalmente independiente de nosotros pero que puede afectarnos causalmente. Los objetos matemáticos, desoyendo lo que, por ejemplo, decía Kurt Gödel, no nos afectarían de esta manera causal y no serían un problema central en este momento. Como espero hacer valer argumentativamente en las próximas secciones me parece que esto no es así.

\subsection{ANTIESENCIALISMO Y PLURALISMO DESCRIPTIVO}

En la formulación de la tesis panrelacionista hemos visto que Rorty introduce la idea de que hay una pluralidad de descripciones de un mismo objeto y que hacemos bien en elegir una de ellas en lugar de otras dependiendo de los objetivos y fines que tengamos a la vista de forma práctica e intelectual. Por tanto, el pluralismo de las descripciones es un corolario que se sigue de, y apoya el panrelacionismo antiesencialista. Es una de sus vertientes. ${ }^{5}$ De hecho, el argumento implícito de Rorty parece ser que donde hay una pluralidad de descripciones es porque hay también una pluralidad de relaciones para describir un objeto y, por lo tanto, ese objeto no tiene ninguna esencia o estructura ensí, y viceversa. Sin embargo, para no caer en el relativismo extremo que diría que estas descripciones son igualmente pertinentes respecto del objeto porque todas ellas son iguales en términos generales -todas son descripciones, por ejemplo, del 17-- Rorty sostiene, como ha hecho también Putnam, que algunas son mejores que otras y que podemos introducir esta diferencia valorativa, supuestamente suficiente para superar el relativismo, en función de los fines a los que sirvan dichas descripciones. En efecto, Rorty considera que todas las descripciones son meras herramientas para lidiar con el mundo pero que unas

${ }^{5}$ He indicado en la nota 3 que hay teorías estructuralista en filosofía de la matemática y en filosofía de la física que postulan relaciones básicas sobre las que se constituyen los "objetos". Nada impide que estas relaciones que conforman las estructuras en las que emergen los objetos sean perfectamente objetivas y no dependan, por tanto, de nuestras descripciones, creencias y deseos. Una ontología semejante puede ser realista en sentido robusto, no antiesencialista, aunque, al mismo tiempo, solo se postule una ontología básica de relaciones. 
serían mejores que otras dependiendo de lo que queramos hacer en cada momento con ellas, del fin para el que las pongamos prácticamente a funcionar (RORTY 2000a, p. 143 y 146). Y, por supuesto, "tanto desde un punto de vista filosófico como desde un punto de vista práctico todos estos objetivos se encuentran en situación de igualdad" (RORTY 2000a, p. 146). Por ejemplo, para enseñar a un niño a contar no se puede empezar diciendo que el 17 es la raíz cuadrada de 289. Pero para saber si alguien ha entendido cómo se hacen raíces cuadradas puedes proponerle calcular la raíz cuadrada de 289 y ver si le da 17. $\mathrm{Y}$, por supuesto, enseñar a contar o enseñar a hacer raíces cuadradas son fines igualmente legítimos.

El pluralismo de las descripciones funciona en Rorty a dos niveles muy diferentes a la hora de reforzar la tesis antiesencialista. En el primer nivel Rorty usa el pluralismo de las descripciones intrarregionalmente, por decirlo clásicamente así. Los ejemplos anteriormente esbozados operan intrarregionalmente. Dentro del ámbito interno de los números naturales o de las concepciones biologicistas y sociales sobre las mujeres podemos ofrecer distintas descripciones de estos objetos. Dichas descripciones lo son de distintas relaciones que confluyen en un nudo al que nos referiremos con el nombre que en cada caso demos al objeto. Estos casos de pluralismo descriptivo interno y panrelacionista, sin embargo, refuerzan de formas desiguales el antiesencialismo. Según el análisis anterior, la pluralidad de descripciones del número 17 no tiene un carácter creativo del objeto, como hemos visto. Más bien el 17 es como el hoyo de un campo de golf ontológico en el que todas esas relaciones confluyen. No por cambiar las descripciones y, por tanto, las relaciones que describimos con ellas, estamos cambiando necesariamente el objeto. Una semántica fregeana inocua podría servir aquí. Tendríamos distintos sentidos para una misma referencia. Además, aunque haya muchas formas de describir las relaciones que confluyen en el objeto $X$ que llamamos " 17 " y el 17 no tenga más consistencia ontológica que la que le viene de prestado por ser el nudo en el que desembocan todas ellas, no por eso nos hemos movido del lugar ontológico X o, en palabras de Rorty, "del centro de gravedad descriptiva" (RORTY 1998a, p. 105) que llamamos "el 17". Sin embargo, hemos visto que con los objetos sociales esto seguramente no ocurra así. Las descripciones posibles de las relaciones sociales en las que intervienen la mujer o, en su caso, tal y como enseña la antropología social (GEERTZ 1973), la familia, el matrimonio o cualquier otra institución social, tienen un carácter creativo en el siguiente sentido. Cambia las descripciones como si las nuevas 
estuvieran ya justificadas, apóyalas con cambios concomitantes en las relaciones sociales reales que esas descripciones expresan lingüísticamente y que ellas permiten también crear y afianzar mediante nuevos vocabularios otra manera de hacer austinianamente cosas con palabras--, y habrás cambiado la esencia de la mujer o de lo que sea. Mejor aún, estarás en vías de mostrar que no hay ninguna esencia que apoye un determinado uso de la palabra "mujer" y unos determinados roles sociales derivados más o menos directamente de esa esencia. Por tanto, si creas progresivamente un contexto previo de nuevas relaciones en las que pensar en, y situar a, ciertos colectivos humanos, entonces concebirás a sus integrantes en "lo que son" de formas diferentes. Son objetos más "líquidos" que pueden diluirse en otras relaciones más creativas y novedosas, quizás con ciertos límites en algunos casos también.

Lo que muestra esta disimetría entre el 17 y la mujer es que el uso intrarregional del pluralismo de las descripciones no lleva directamente y sin más matizaciones al antiesencialismo ontológico. Seguramente entre los extremos que representan el 17 y la mujer haya otros objetos de otras regiones ontológicas que cubran un espectro más o menos continuo en cuanto al grado de vinculación que se da en ellos entre el panrelacionismo ontológico, como trasfondo del antiesencialismo, y el pluralismo descriptivo. ${ }^{6}$ Lo que estoy sugiriendo es que podría muy bien suceder que, en contra de la línea argumental principal de Rorty, no siempre que hay pluralidad de descripciones y de formas relacionales diversas de caracterizar un objeto, estamos ante un caso semejante de antiesencialismo. Hay que estudiar pormenorizadamente caso por caso empezando por la misma matemática y sus distintas áreas, especialmente la geometría y la aritmética, y recorriendo, en un posible programa de investigación, todas las ciencias y demás discursos

Hay, sin embargo, un segundo nivel en el que Rorty usa transregionalmente el pluralismo de las descripciones para afianzar la idea de que el mundo no tiene esencia. Ahora no se trata de que los objetos particulares que conforman alguna región circunscrita no tengan esencia, sino de que el mundo en su conjunto no tiene tampoco ninguna. Además, en este caso de extrema generalización no se trata de que concluyamos la falta de esencia del mundo a

\footnotetext{
${ }^{6}$ Como hipótesis de trabajo la idea de que hay un espectro más o menos continuo entre dos extremos, como los señalados aquí, es una forma abierta y generosa de plantear la cuestión. Sin embargo, bien podría suceder que se viera a la postre que estamos ante una dicotomía sin términos intermedios.
} 
partir del sumatorio de la falta de esencia de cada una de sus posibles regiones ontológicas. Por el contario, hay, como mostraré, un salto considerable en la argumentación cuando se ingresa en este nivel transregional.

El uso transregional del pluralismo de las descripciones para afianzar el antiesencialismo se manifiesta en todos aquellos numerosísimos ejemplos de Rorty en los que muestra que distintas motivaciones y fines pueden hacer que describamos interpretativamente de formas muy diferentes no ya los objetos, más o menos novedosos, que caen dentro de un ámbito ontológico, sino el mundo en su conjunto. Rorty sostiene que, por ejemplo, la descripción de los objetos en términos de la física de partículas elementales es útil para determinados propósitos en virtud de los cuales se "puede contribuir a lograr nuevos avances tecnológicos o [...] imaginativas redescripciones del mundo como un todo" (RORTY 2000a, p. 153). Pero la descripción de un objeto en estos términos no es más verdadera que en términos de nuestro vocabulario cotidiano o en términos de un vocabulario "mitológico", sea religioso o metafísico, porque el mundo - no ya el 17 o las mujeres u objetos como ellos-no tiene ningún en-sí del cual se dé mejor cuenta desde un lenguaje que desde otro. Podemos decir que un objeto $X$ es, por ejemplo, una masa de partículas subatómicas, un producto de la evolución de las especies, nuestro vecino de la puerta de al lado o nuestro hermano en el reino de Dios. Todo depende del fin para el que pongamos a funcionar prácticamente ese vocabulario. Es decir, no se trata ahora de que describamos el 17 o a las mujeres de formas diferentes en función de los fines que tengamos sino de que pongamos a funcionar un determinado vocabulario como un todo para describir el mundo como un todo en función de fines, por decirlo así, de rango superior dando lugar a descripciones plurales del mundo que, para Rorty, también son todas igualmente legítimas.

Por supuesto, Rorty reconoce que puede haber conflictos muy serios que se originan cuando distintos fines y sus vocabularios anexos dan lugar a prácticas y visiones del mundo en su conjunto muy opuestas, incluso contradictorias entre sí. Cuando se llega a este punto hay que tomar decisiones racionales de tipo heurístico y práctico. Podemos, por ejemplo, prescindir, o intentar prescindir, de un tipo de vocabulario y de las descripciones del mundo que hacemos con él mediante estrategias eliminativistas o reduccionistas. Podemos intentar aplicar una estrategia de jerarquización de vocabularios en nuestra visión general del mundo de tal forma que si un vocabulario de orden inferior entra en conflicto con uno de orden superior tengamos siempre a 
mano un criterio normativo para determinar cuál de ellos es mejor mantener para preservar nuestra visión del mundo y su equilibrio global. Podemos también ensayar y mantener una estrategia de compartimentalización de vocabularios y descripciones. En este último sentido Rorty sostiene que, mediante una estrategia de compartimentalización de las creencias, es decir, de los fines a los que sirven esas creencias, una persona puede ser, sin contradicciones, profesora de biología evolucionista durante la semana e ir a misa los domingos (RORTY 2000c, p. 79). Sin embargo, ninguno de esos vocabularios se acerca más a la realidad del objeto - por ejemplo, a la hora de decir cómo han llegado a existir los seres humanos y qué es un ser humano-que a su mera apariencia. Eso sí, los proyectos a los que se supeditan esos distintos vocabularios y que les otorgan distintos valores de utilidad pueden ser incompatibles entre sí hasta el punto de hacerse irrelevantes unos para otros. "La ciencia física es sencillamente otro proyecto humano más que, como todos los proyectos humanos, puede eclipsar la posibilidad de otros proyectos incompatibles con él" (RORTY 2000a, p. 156). Esta posibilidad, como hemos visto, no es automática para Rorty, pues siempre podemos aplicar a nuestro sistema de creencias una estrategia de compartimentalización, como ya hizo Wittgenstein y otros muchos antes que él con las creencias científicas y religiosas, y solventar así el problema.

De la argumentación precedente podemos concluir que Rorty cambia radicalmente de problemática cuando pasa del pluralismo de las descripciones en su uso intrarregional a su uso transregional. Una cosa es sostener que podemos describir múltiples relaciones que confluyen en un $X$--creando incluso en algunos casos un objeto nuevo-- y que esas descripciones son mejores o peores dependiendo de nuestros fines particulares, y otra muy distinta es afirmar que estaremos frente a mundos poblados de objetos muy diferentes cuando establezcamos los fines generales en función de los cuales hacemos una determinada descripción y elegimos el vocabulario adecuado para hacerla". En este último caso el "mundo" no tendría esencia, no tendría ningún en-sí porque comparece y se consolida ante nosotros de formas cambiantes y plurales dependiendo del fin gracias al cual seleccionamos un determinado vocabulario, hacemos nuestras descripciones en función de él y ponemos en marcha unas determinadas prácticas para lidiar con el mundo visto

\footnotetext{
7 Por ejemplo, Jean-Pierre Cometti ha incurrido recientemente en esta misma confusión (COMETTI, p. 157).
} 
precisamente a través del prisma que proporcionan este vocabulario y estas prácticas. Pero esto último no es exactamente lo mismo que el panrelacionismo antiesencialista del que se partió sino algo más próximo a un idealismo descriptivo para el cual el mundo en su conjunto brota o se constituye de distinta manera en función de las descripciones interpretativas que hagamos de él. En definitiva, el uso transregional del pluralismo de las descripciones, asociado a determinados vocabularios omniabarcantes para describir el mundo, daría lugar a una tesis antiesencialista. Sin embargo, no se habría llegado a ella a través del panrelacionismo pues, como he indicado, no se trataría ahora de que haya un haz de relaciones cuyos nodos constituyen los objetos, sino de la elección de unos fines, de unos vocabularios y de unas prácticas que abren el mundo ante nosotros de formas muy diversas e, incluso, incompatibles entre sí. Esta situación da lugar al problema que trataré a continuación, a saber, el de indagar cómo afectan estas tesis de Rorty y las críticas que propongo a la comprensión de la independencia causal del mundo.

\section{TRIANGULACIÓN SUJETO, MUNDO, CREENCIAS}

La conclusión antiesencialista de conjunto que extrae Rorty del argumento panrelacionista, según la cual el mundo no tiene ningún en-sí, podría hacer peligrar la tesis realista de la independencia causal del mundo respecto de nosotros y su compresión y efectividad filosófica plena. Este peligro procede de dos frentes relacionados. Por una parte, si algo no tiene ningún en-sí porque su constitución como objeto depende de la red de relaciones en la que nosotros, en función de intereses variados, lo emplazamos, entonces hay que explicar qué independencia causal tiene respecto de nosotros, cuál es esta causalidad independiente y cómo opera. Y esto solo se puede hacer si, al menos, explicamos cuáles creemos que son los lazos causales concretos que puede generar con nosotros eso que no tiene ningún en sí y que se ha constituido como rendimiento de nuestros usos socio-lingüísticos. El mundo podrá ser independiente causalmente de nosotros pero esto no significaría filosóficamente nada, o muy poco, si no somos capaces de decir, según nuestras creencias, si su estructura causal es una u otra, aunque esta estructura causal sea sensible al contexto experimental como sucede con los objetos cuánticos. No es lo mismo decir que una persona está poseída por el diablo que decir que sufre una enfermedad o un desorden neurológico. Por otra parte, la reflexión previa sobre el antiesencialismo ha puesto de manifiesto que, 
dependiendo de cómo describamos el mundo desde el vocabulario global que hayamos elegido o se nos haya impuesto socio-culturalmente, parece que, por el hecho de hacerlo así, estamos pensado también que el mundo es el responsable causal de que lo describamos interpretativa y justificadamente de esa manera. Sin esta creencia de base no pensaríamos que el vocabulario usado fuera especialmente útil. Los distintos vocabularios implican distintas creencias de base, en el sentido de una Urdoxa que opera en el trasfondo de toda otra creencia, acerca de la estructura causal del mundo tal y como, por ejemplo, los vocabularios en los que aparecen diablos o, por el contrario, aparecen trastornos neuronales ponen de manifiesto. Por eso los distintos vocabularios son útiles a distintas comunidades e individuos. Pero esta variedad de creencias básicas acerca de la estructura causal del mundo supondría que el mundo respaldaría causalmente casi cualquier descripción que hagamos de él del mismo modo que las múltiples relaciones que vierten sus aguas en el 17 o en la mujer respaldan la pluralidad de descripciones interpretativas que generemos. El pluralismo de las descripciones, sobre todo en su vertiente transregional, afianzaría distintas creencias de partida sobre cuáles son los lazos causales entre el mundo y el resto de nuestras creencias y cómo describir esos lazos causales. Esta situación supondría inmediatamente que el nexo causal entre el mundo y nosotros podría ser demasiado débil como para aceptar realmente su independencia causal. Tendríamos la independencia causal del mundo, pero ¿de qué mundo? ¿Un mundo con íncubos y súcubos? Aparecería aquí una nueva versión del mundus absconditus que emergería ahora no tanto porque exista un telón de fondo insalvable de conceptos y creencias del que no podemos prescindir cuando describimos interpretativamente el mundo y que nos llevaría al escepticismo agnóstico, sino porque los lazos causales mundo/creencia se habrían difuminado tan extraordinariamente, dada la enorme pluralidad de posibilidades de descripción de estos lazos causales, que no habría motivos para hablar en concreto de la independencia causal del mundo a nos ser que lo hiciéramos de una forma nouménica kantiana, que Rorty siempre rechaza, como el $X$ que causa todo tipo de pluralidad de creencias y descripciones legítimas dadas históricamente o todavía por llegar imaginativamente.

Rorty cree posible solucionar este problema cuando sostiene que "temeremos que el mundo esté a punto de esconderse siempre que pensemos que las conexiones causales con el mundo no son una vía suficientemente fuerte de establecer lazos de unión con él" (RORTY 2000d, p. 124). Pero, como he indicado, el problema ahora no es sólo ontológico, no es, por tanto, si hay 
lazos causales de unión suficientemente fuertes entre el mundo y nosotros, sino también epistemológico, es decir, la dificultad residiría en saber cuáles son estos lazos causales fuertes cuando, para contestar esta cuestión, no podemos contar con el punto de vista del ojo divino y cuando, además, estamos anclados en esquemas conceptuales y juegos de lenguaje previos todos los cuales, según Rorty, van a constituir las relaciones dentro de cuyos cruces vamos a emplazar "líquidamente" los objetos y los vamos a pensar con nuestras creencias y conceptos. ¿Estamos hablando de la fortaleza de la relación causal del diablo con nosotros o de la fortaleza de las relaciones causales de los procesos cerebrales con nuestros comportamientos? Además, la pluralidad de objetivos para describir el mundo y la selección concomitante de diferentes conceptos para llevar a cabo esta descripción contextualizada no mejora sino que empeora aún más la situación, dado que nuestro acceso a diferentes posibilidades de describir los nexos causales entre el mundo y nosotros viene determinada en gran medida por los esquemas conceptuales que ponemos previamente en juego para atender determinados fines.

Adviértase, sin embargo, que con estas afirmaciones no estoy diciendo que todas nuestras creencias sean acerca de las relaciones causales que las producen o que todas nuestras creencias describan esos nexos causales, aunque, por supuesto, haya creencias también acerca de dichos nexos causales. Nada de lo que estoy proponiendo tiene estas implicaciones como tampoco implica una confusión entre causas y razones. Simplemente estoy indicando que el nexo causal concreto que creamos que hay entre el mundo y nosotros dependerá de los juegos lingüístico-culturales previos que determinen nuestras descripciones y que, dada la pluralidad de vocabularios y descripciones, esta situación deja en el nimbo la comprensión concreta de la independencia causal del mundo pues habría muchas independencias causales de muchos mundos distintos e incompatibles entre sí.

De una forma muy interesante Rorty liga esta cuestión referida a nuestros lazos causales con el mundo al problema de la triangulación que planteó Davidson, a quien Rorty parece seguir en este punto (DAVIDSON 1990). Rorty extrae ciertas consecuencias del hecho de que exista una triangulación causal entre el individuo, sus pares culturales -su ethnos-- y el mundo. La primera de ellas es la siguiente:

"La coherencia [de las creencias de un sujeto], la verdad [de esas creencias] y la comunidad [el hecho de que nuestros pares culturales consideren 
generalmente coherentes nuestras creencias] van juntas, y no porque la verdad se defina en términos de coherencia en lugar de hacerlo en términos de correspondencia; en términos de prácticas sociales en lugar de hacerlo en términos de lidiar con fuerzas no humanas, sino simplemente porque adscribir una creencia es automáticamente adscribirle un lugar en un conjunto ampliamente coherente de creencias la mayor parte de las cuales son verdaderas" (RORTY 2000b, p. 16).

A la hora de interpretar qué significa en este texto "la verdad" de nuestras creencias debemos tener en consideración la siguiente observación. Sabemos que Rorty acepta como legítimo el uso precautorio del término "verdad" en determinados contextos (RORTY 1991d, p. 128 y 2000b, p. 4). Dada la sana conciencia de nuestro falibilismo cognitivo que nos impide ser parroquianos de nuestras propias creencias (MCDOWELL 2000 115), es conveniente decir siempre que una creencia puede está bien justificada pero que podría no ser verdadera. Este uso precautorio significa, por tanto, que una creencia puede estar justificada a la luz de los estándares normativos temporales de un grupo humano y, sin embargo, no estar justificada para un auditorio futuro. Podemos, pues, intentar asegurarnos en diversos grados de justificar nuestras creencias ante nuestros iguales pero no de que sean verdaderas en un sentido "esencialista" según el cual habríamos logrado cancelar de una vez por todas el uso precautorio del predicado "...ser verdadera". Es decir, nunca podremos decir con certeza que nuestras creencias no necesitarán cambios ni retoques posteriores en su contenido, según los proponga cualquier generación posterior más capaz o más imaginativa que nosotros, porque son definitivamente verdaderas. ${ }^{8}$ Si tenemos en cuenta este uso precautorio del concepto de verdad, entonces podemos entender por qué Rorty, poco antes de la cita anterior, había dicho lo siguiente:

"[L]a mayoría de nuestras creencias deben estar justificadas a los ojos de nuestros pares $[\ldots]$ Si nuestras creencias no estuvieran justificadas - si nuestros pares no nos atribuyeran una red de creencias y deseos ampliamente coherente-tendrían que concluir que o bien nos habían entendido mal o bien no hablaban nuestro lenguaje" (RORTY 2000b, p. 16).

\footnotetext{
${ }^{8}$ Una discusión muy útil acerca de la raigambre deweyana de estas tesis falibilistas de Rorty y también acerca del distinto modo en que Rorty y Putnam se apropian de ellas y las desarrollan puede encontrarse en (FAERNA 2014).
} 
Rorty, por tanto, puede pasar, aparentemente sin problema alguno, de un texto donde habla de la justificación a uno donde habla de la verdad de nuestras creencias. ${ }^{9}$ No voy a bucear ahora en las complicaciones, sutilezas y consecuencias que se encierran en el manejo que hace Rorty de los términos "verdad" y "justificación", algo que ya he hecho en otro lugar (GONZÁLEZCASTÁN 2014). Lo que sí es importante hacer es explicitar mejor cómo afecta este planteamiento de la triangulación que hay entre (1) la coherencia de nuestras creencias, (2) su justificación/verdad y (3) la comunidad que adscribe coherencia y justificación a dicha creencia con el problema de los lazos causales entre un mundo antiesencialista y nuestras creencias. ${ }^{10}$ La vía más directa para hacerlo es diciendo que considerar que nuestras creencias están justificadas implica, al menos, una atribución temporal, que puede ser histórica y biográficamente más o menos dilatada, de determinadas expectativas comunitarias acerca de cómo se van a explicar los lazos causales que hay entre determinadas creencias y el mundo. Vuelvo a insistir en que esto no significa que todas nuestras creencias sean acerca de estos lazos causales, sino que en nuestro sistema doxástico habrá también creencias acerca de cuál es el vocabulario adecuado para hablar de estos lazos causales y cuáles son en concreto. Cuando alguien dice: "mira, allí hay una vaca" y esa misma persona, como científico, afirma: "aquí hay un electrón porque hay determinadas marcas

9 Como muy bien indica críticamente Bilgrami, siguiendo a Putnam, tener en cuenta la expresión cautelar “... y no ser verdad” indica que nunca es una cuestión baladí preguntarse si una creencia que se considera bien justificada es, sin embargo, verdadera; verdadera en un sentido no precautorio (BILGRAMI 2000, p. 244). Davidson, en sus desacuerdos con Rorty, interpreta esta distancia entre verdad y justificación en un sentido objetivista que quedaría diluido o eliminado, según Davidson, con el mero uso precautorio propuesto por Rorty. Davidson sostiene que la verdad es objetiva en el sentido de que una creencia será verdadera independientemente de que esté justificada por toda nuestra experiencia disponible o por la de cualquier otra generación futura. Toda experiencia fruto de la investigación siempre será limitada (DAVIDSON 2000, p. 67). Además, Davidson defiende lo siguiente: "Es posible tener una creencia solo si sabemos que las creencias pueden ser verdaderas o falsas. Puedo creer que ahora está lloviendo, pero esto es así porque sé que si está o no está lloviendo no depende de que yo lo crea o de que lo crea todo el mundo o de que sea útil creerlo [...] La correspondencia [de nuestras creencias con el mundo], a la vez que es una noción vacía como definición, sin embargo capta el pensamiento de que la verdad depende de cómo sea el mundo, y esto debería ser suficiente para desacreditar la mayoría de las teorías epistémicas y pragmatistas" (DAVIDSON 2000, p. 72).

${ }^{10}$ Un filósofo que ha trabajado con profusión el problema de la triangulación en Rorty y Davidson es B. Ramberg (cf. RAMBERG 2014 y 2011). 
en esta cámara de niebla", y las comunidades relevantes de pertenencia sostienen que estas creencias son coherentes y justificadas, lo que estas comunidades están sosteniendo en el fondo es que ellas y ese individuo creen justificada, aunque cautelarmente, que el mundo, en concreto una vaca y un electrón, han sido en parte causalmente responsables de su creencia en la existencia de esas entidades.

Sin embargo, el antiesencialismo panrelacionista de Rorty, como deja la puerta enteramente abierta a una pluralidad de vocabularios y de descripciones del mundo igualmente adecuados (busca un fin adecuado y tendrás un vocabulario adecuado para describir el mundo de acuerdo con este fin), también permite que distintas comunidades -que viven o no en un mismo tiempo histórico-consideren coherentes y justificadas distintas creencias, incluso cuando estas creencias son incompatibles entre sí. Pero esto, de nuevo, significa que la independencia causal del mundo es una expresión vacía porque no hay forma neutral de determinar de qué estamos hablando como siendo causalmente independiente respecto de nosotros. Por ejemplo, la disputa entre darwinistas y creacionistas dentro de una misma sociedad en sentido amplio (la estadounidadense, la occidental, etc.) es fundamentalmente una disputa acerca de la causa o causas de nuestra actual constitución biológica. En este caso, sostener que el mundo es causalmente independiente de nosotros es una afirmación que, parafraseando a William James, no se puede cambiar por ningún valor concreto. Por tanto, también la idea de "independencia causal del mundo" hay que ponerla a funcionar, según el motto de la VI conferencia de James sobre pragmatismo, para darle cuerpo y consistencia (JAMES 1907/1975). Pero en el mundo antiesencialista y panrelacionista de Rorty en el que se da por válidas una pluralidad indefinida de vocabularios y descripciones, en cuanto se pone a trabajar esta idea de la independencia causal del mundo no hay forma concreta de dotarla de significado o, mejor dicho, tiene tantos que no sabemos qué es lo que se supone que es independiente de nosotros. Incluso podríamos preguntarnos, también en el más puro espíritu pragmatista jamesiano, qué diferencia en la práctica se obtendría de mantener la independencia causal del mundo respecto de nosotros. Parece que ninguna. Lo que importa, de acuerdo con la tesis de la triangulación, es que nuestras creencias sean coherentes y que la justificación que demos de ellas esté avalada por nuestra comunidad de referencia. Pero esto lo pueden lograr muchos sistemas distintos de creencias que caracterizarían de formas diversas e 
incompatibles aquello que las comunidades que sostienen esos sistemas de creencias consideran causalmente independiente de sus creencias y deseos.

Me parece que esta consecuencia es, al menos en parte, fruto del giro lingüístico que abraza Rorty. Como es sabido Rorty adopta el punto de vista de Sellars de que las creencias se mueven exclusivamente en el "espacio lógico de las razones". Esto significa que ninguna creencia, ya sea verdadera o falsa, es otra cosa que un asunto lingüístico ni expresa otra cosa que sus relaciones con otras partes del lenguaje. Lo único que puede verificar una proposición es otra proposición. En ningún caso, pues, una creencia será verdadera "extralingüísticamente" o por motivos extralingǘsticos, es decir, porque describa, por ejemplo, lo que la comunidad que la sostiene cree que es su causa en el mundo. Esta versión rortyana de la tesis de la autonomía de la gramática de Wittgenstein serviría de justificación de por qué distintas comunidades de sujetos determinarían de formas plurales -formas que se no se pueden unificar de momento-- los elementos del mundo que consideran causalmente independientes de sus creencias y deseos.

Es conocido que uno de los elementos fundamentales que sirven a Wittgenstein para romper amarras con el positivismo lógico y con sus propias tesis del Tractatus acerca del isomorfismo entre el lenguaje y el mundo es la tesis de la autonomía de la gramática que empezó a desarrollar después de su regreso a Cambridge en enero de 1929. Ahora las proposiciones significativas no lo son porque sean un espejo o porque compartan la estructura lógica de un posible estado de cosas del mundo. No hay semejante isomorfismo estructural entre el mundo y el lenguaje. Por el contrario la gramática del lenguaje y de los distintos lenguajes es auto-contenida y completa. Esto significa que el conjunto de reglas que operan en el uso correcto de un término o una expresión no están determinadas por hechos mundanos de ningún tipo o por la estructura lógica de estos hechos. En este sentido, la gramática es arbitraria y libre respecto de cualquier lazo con la realidad. Tampoco es corregible o susceptible de mejora por consideraciones filosóficas de ningún tipo sobre las relaciones entre el mundo y el lenguaje. Como se ve Rorty extiende ahora esta independencia de la gramática a la independencia de las creencias y de su verdad o justificación respecto de la independencia causal del mundo. Pero, como estoy tratando de argumentar, este hecho vuelve ininteligible el modo en que podemos hacer efectiva la independencia causal del mundo.

En definitiva, decir vagamente que hay una conexión causal suficientemente robusta con el mundo que impide que lo consideremos como 
mundus absconditus (RORTY 2000d, p. 124) no ayuda mucho para que el mundo no tenga esta característica de poder ocultarse a nuestras creencias. Es desde estas creencias desde las que hacemos epistémicamente concretos los lazos causales del mundo que nos están afectando porque podemos establecer descriptiva y teóricamente cuáles son exactamente $\mathrm{y}$, por lo tanto, de qué mundo estamos hablando. Por tanto, el problema del mundus absconditus se plantea otra vez dada la mediación conceptual y cultural entre nuestras creencias y descripciones y el mundo. Apelar, como hace Rorty y en los términos que él lo hace, al argumento de la triangulación de Davidson no resuelve nada. Dada esta incómoda situación se desvanece la robustez del lazo causal mundo/creencias el cual, aunque haya que suponerlo desde nuestras intuiciones realistas de sentido común más básicas y contumaces, no pasa de ser absolutamente indeterminado y sin contenido delimitado y efectivo, porque su determinación concreta pasa precisamente por el establecimiento de las propias creencias desde las que articulamos lingüísticamente cuáles son los objetos que nos afectan causalmente.

Por tanto, decir que hay lazos causales sólidos entre el mundo y las creencias es como situar un objeto detrás de distintos cristales con diversos grosores, texturas, colores y grados de distorsión luminosa gracias a los cuales lo vemos de muy diferentes maneras, como si fuera incluso muchos objetos diferentes. El lazo causal entre el objeto y nuestras descripciones será todo lo robusto que queramos, pues ciertamente puede haber una covariación causal entre cambios en el objeto y cambios en nuestra descripción de él, pero no tenemos ni idea de qué objeto realmente produce estas descripciones dado que produce una pluralidad de ellas muy diferentes y hasta contrarias pero todas igualmente legítimas. Todo depende de si las queremos para hacer ciencia o para consolarnos de este valle de lágrimas o para lo que sea. En otras palabras, el mundo necesitará tener tantas capacidades causales, por así decir, como ámbitos de descripción igualitaria posible hayamos establecido previamente y nos interesen individual y colectivamente de modo tal que no habrá manera de saber en qué consiste la supuesta y genuina independencia causal del mundo pues el mundo responderá dócilmente en muchos casos a nuestros diversos intereses y los respaldará causalmente de formas tan variadas como variados sean estos intereses previos.

Cierto es que intervenimos en el mundo con nuestra acción, pero no menos cierto es que intervenimos con determinadas acciones y no con otras dependiendo de las descripciones concretas que hacemos de él, las cuales, 
como implicaciones suyas, incluirán qué efectos causales esperamos razonablemente del mundo. Además Rorty sostiene lo siguiente:

"No hay ninguna verdad ineludible que los metafísicos o los pragmatistas estén procurando evitar o captar, pues cualquier candidato a la verdad puede ser eludido mediante la elección de una descripción adecuada [alternativa], o respaldada mediante otra elección semejante" (RORTY 2000a, p. 156).

Por tanto, parece que el relativismo no se ha esquivado del todo y que la independencia causal del mundo está en entredicho. Cambia el lenguaje y cambiarás, de una manera sólida e inapelable, la descripción adecuada del mundo que está conectado causalmente contigo y las prácticas anexas a esas descripciones

\section{RORTY: CAUSACIÓN Y CREENCIA}

Dado el problema del debilitamiento e indeterminación de los lazos causales mundo/creencia y supuesta la tesis de la mezcolanza y sus derivaciones antiesencialistas, debemos afrontar finalmente una nueva dificultad que surge del modo en que Rorty explicita la independencia causal del mundo. Con esta dificultad culmina la imposibilidad de hacer un todo coherente de muchas de las tesis de Rorty.

Dado su panrelacionismo de base y su pluralismo descriptivo, sorprende mucho que la división ontológica fundamental que propone Rorty es la que se da entre aquellos objetos

"que nos hacen tener creencias sobre ellos por medios causales bastante directos y otros objetos. En el caso de este último tipo de objetos, las relaciones causales relevantes son $\mathrm{o}$ bien terriblemente indirectas $\mathrm{O}$ sencillamente inexistentes [...] La explicación de cómo se han llegado a causar en nosotros las creencias y deseos sobre la felicidad, la castidad o la voluntad de Dios no nos devolverá a estos objetos" (RORTY 1991c, p. 106).

Desde luego, lo que sorprende de esta cita es, como he indicado, saber cómo se aviene con el panrelacionismo y, si mi crítica en la sección anterior está bien hilada, su dificultad máxima consistiría en compaginarla con la independencia causal del mundo cuando sabemos que esta independencia se constituye y materializa de formas muy plurales según nuestros equipamientos justificativos socio-lingüísticos. Según este pluralismo, que revierte, como hemos visto, en una pluralidad de formas en que concebimos los humanos el 
mundo como causalmente independiente, es difícil hacerse cargo de la distinción ontológica que Rorty acaba de plantear. Como esta dificultad parece clara, en lo que sigue me centraré en escrutar el alcance de esta propuesta de Rorty considerada en sí misma. El problema que quiero tratar ahora es, por tanto, cómo se puede saber que hay tales relaciones causales y cuáles son en concreto cuando, sin embargo, partimos de una situación en la que no podemos tener ninguna idea o creencia particular sobre el mundo, ni siquiera las creencias perceptivas, que no sea la de su mixtura con lo que aportan las distintas tradiciones y sus conceptos a los individuos que van ingresando en ellas y cuando, además, el mundo respalda causalmente multitud de descripciones posibles de él dado el panrelacionismo transregional.

Sin embargo, antes de indagar críticamente en estas cuestiones debemos aclarar la noción de objeto que maneja Rorty. Este filósofo habla de objetos en un sentido muy amplio. "Todo lo que se necesita para que una cosa sea un objeto es que se hable de ella de una forma razonablemente coherente" (RORTY 1999, p. 85). La felicidad, la castidad, Dios y su voluntad, pero también el número pi, las supercuerdas, el flogiston, los derechos humanos, las mujeres, los varones o los Reyes Magos son, en este sentido genérico, objetos porque de ellos poseemos conceptos gracias a los cuales formamos discursos variados, gestados históricamente, que son más o menos razonables, complejos, coherentes y envolventes. En general, estos conceptos, así como las oraciones y los discursos en los que ellos se insertan, producen en quienes los tienen la sensación de que, con ellos, se está hablando acerca de algo y refiriéndose efectivamente a ello, así como a sus propiedades y relaciones, en un vuelo que, justificada o injustificadamente, pretende ir más allá del sistema de creencias propio y de la mezcolanza del mundo con él para plantarse en el mundo tal y como es en-sí.

Esta noción de objeto es cercana a la de objeto intencional de la fenomenología. Incluso Rorty lo reconoce explícitamente: "Así pues, la única noción de objeto que necesitamos es la de 'objeto intencional'. Un objeto intencional es aquello a lo que se refiere una palabra o descripción" (RORTY 1991c, p. 106). En otros términos, objeto "intencional" es aquel sentido que es comprendido con mayor o menor grado de claridad por un sujeto o grupo de ellos, un sentido sobre el que se puede trenzar un discurso más o menos coherente y ciertas prácticas concomitantes. Este discurso ofrece un contexto contingente para recontextualizar nuevas creencias sobre dichos objetos y para que el contacto causal del mundo con nosotros produzca creencias en nuestro 
sistema doxástico, pues son esos discursos los que nos han "programado" para poder hablar sobre el mundo ${ }^{11}$.

Ahora que tenemos a la vista la noción de objeto que maneja Rorty es conveniente analizar la potencia del criterio que ha proporcionado para distinguir unos objetos de otros dadas todas sus tesis anteriores. Creo que este criterio nos obligará a ir más allá de la noción de "objeto intencional". El criterio, recordémoslo, consiste en discernir si la explicación de cómo se ha causado en nosotros la creencia en determinados objetos nos retrotrae de nuevo a estos objetos o no lo hace. Más exactamente, lo que afirma Rorty con su criterio es que, para muchos conceptos y para las creencias que se arremolinan en su entorno, hay en algunos casos, e independientemente de las explicaciones o interpretaciones que propongamos, caminos de vuelta desde las creencias a los objetos del mundo que las han causado, mientras que en otras ocasiones no hay tales caminos de retorno. Por tanto y sólo para aclararnos, podemos nosotros, aunque no lo haga así Rorty, llamar a los objetos que existen al margen de su carácter intencional, es decir, al margen de ser los "referentes" de los discursos reales que produce una comunidad de hablantes en función de sus trasfondos históricos y culturales, objetos reales. Si adaptamos, creo que sin ninguna violencia, su forma de hablar podemos decir que un objeto es real cuando hay un camino causal que podemos desandar y que une nuestra creencia con el objeto porque previamente el objeto ha "abierto" un camino causal desde sí mismo hacia nuestra creencia. Inversamente, un objeto es inexistente cuando esto no sucede así como pasa, según los ejemplos de Rorty mencionados anteriormente, con Dios o la felicidad o lo que sea. En estos casos nunca encontraremos el objeto de la creencia al final del camino sino sólo un entramado de discursos basados en ciertos conceptos, en ciertos sentidos, que ofrecen, como mucho, una

11 "El mundo -dice Rorty-no habla. Solamente nosotros hablamos. El mundo puede, una vez que nos hemos programado a nosotros mismos con un lenguaje, causar que sostengamos determinadas creencias" (RORTY 1989, p. 6). El mundo es, por decirlo así, un catalizador para creer cosas muy variadas y hasta opuestas sobre él y, por lo tanto, para generar una pluralidad de descripciones legítimas que están en función de los contextos lingüísticos y relacionales y de los fines que tengamos en cada ocasión. Como las creencias son diferentes en función de las diferentes tradiciones a las que cada cual pertenezca, así también serán diferentes las creencias que el mundo cause en los individuos pertenecientes a cada una de estas tradiciones y las descripciones que hagamos de él, las cuales son, a la vez, subsidiarias y creadoras de las relaciones que caracterizan a los objetos. 
direccionalidad hacia un presunto objeto real, una "referencia", sin ofrecer garantía alguna de que tal objeto exista. Podemos decir también que en la formulación lingüística de las creencias referidas a estos objetos aparecen nombres, conceptos, a los que no les corresponde un objeto real, sino sólo intencional. A este tipo de conceptos podemos denominarlos "conceptos materialmente falsos", por usar la forma de hablar que Descartes utilizó en la tercera Meditación para calificar a aquellas ideas que representan lo que no es nada como si fuera algo. ${ }^{12}$ En el caso de Rorty, como en el de Descartes, surgiría entonces el problema de saber qué conceptos o ideas tienen falsedad material y qué ideas y creencias son materialmente verdaderas. El problema ahora no es el criterio sino las condiciones o reglas de su aplicación que nos permitan saber cuándo hay o cuándo no hay camino de retorno de la creencia al objeto que la causa, sobre todo en casos difíciles. Así pues, ¿de qué depende que se declare que en el camino de vuelta se va a encontrar o no el $X$ que produjo en nosotros la creencia en su existencia porque, en cada caso, hay o no hay tal lugar de partida?

Las dificultades con las que se topa en este punto el panrelacionismo antiesencialista, el pluralismo descriptivo y el realismo de la mezcolanza de Rorty son insuperables, según me parece. Puesto que siempre hay presente objeto intencional, esto es, discurso sobre él y, para la mayoría de las personas, una intención de mentar algo más allá del propio sistema solidario de creencias, da la sensación de que la única manera de acceder al objeto real es a través de nuestra malla de creencias en la que irremediablemente estamos presos y que es lo único con lo que contamos, además de su utilidad práctica, muy variada en función de nuestros intereses. La imposibilidad viene precisamente porque estamos presos en nuestras creencias etnocéntricas y en nuestros modos etnocéntricos de justificarlas que proporcionan interpretaciones funcionales del mundo y que sólo rinden un mundo-en-mezcolanza, en este caso el "nuestro". En estas circunstancias, donde el individuo vive en su máquina tricotosa para trenzar creencias (RORTY 1991c, p. 93), cualquier intento de desandar el camino causal de vuelta para declarar que se ha vuelto efectivamente sobre los pasos que el objeto había abierto hasta nuestra creencia es una petición de

12 Por supuesto, podemos muy bien suponer que Rorty no admitiría la jerga representacionalista, pues la ha criticado por activa y por pasiva como escoria filosófica y rémora de la modernidad. Pero lo que estoy planteando no depende de la discusión en torno al representacionalismo sino de la posibilidad de que Rorty plantee la división ontológica que estamos discutiendo. 
principio manifiesta, pues no puede saber si lo hay o no. Sólo se puede hacer tal cosa si ya se cree que hay tal camino. Pero creer que lo hay sólo puede ser fruto de las explicaciones interpretativas que nuestro sistema de creencias es capaz de generar en función de su dotación doxástica de partida y de los medios contingentes de justificación con los que cuenta. Si tenemos en cuenta, además, que viviríamos en un mundo antiesencialista que no delimita ni impone ninguna descripción de él en su conjunto por encima de otras, entonces se nos enreda todavía más la posibilidad de dar sentido a esta práctica de determinar si los objetos causan en nosotros creencias y si estos medios causales son más o menos directos.

Es cierto que un realista científico no muy exagerado podría apelar a la capacidad de predicción y comprobación a escala humana que tienen algunas de nuestras creencias para sostener que si se dan ambas cosas, entonces es que estamos en contacto cognitivo con la realidad o, al menos, que nos estamos acercando a un conocimiento no interpretativo de la realidad o, en última instancia, que lo que decimos no es puro capricho. Sin embargo, Rorty le corta las alas inmediatamente a este iluso cuando pregunta retadoramente lo siguiente: "¿qué tiene de especial la predicción y el control? [...] ¿Cuál es la relación entre facilitar la predicción y el control y ser 'no en perspectiva': o 'independiente de la mente?" (RORTY 1991a, p. 58). Rorty no encuentra ninguna relación. Eso no quiere decir que podamos llamar ciencia a una actividad humana que no consiga predecir y controlar. La predicción y el control son criterios pragmáticos para discernir lo que es ciencia pero no son criterios de ningún tipo para tener la esperanza racional de que estamos más cerca de algunos rasgos del mundo que cuando no conseguíamos ese control y predicción. Por tanto, la predicción y el control, es decir, cierto tipo de utilidad, no nos sirven para desandar el camino causal de regreso de nuestras creencias a los objetos.

\section{CONCLUSIÓN}

En definitiva, las tesis de la mezcolanza mundo/creencia y el panrelacionismo antiesencialista, junto con sus consecuencias, ofrecen obstáculos muy variados y difíciles de salvar cuando se las quiere compaginar con la tesis de la independencia causal del mundo, tal y como he intentado explicitar en todas estas reflexiones desde distintas perspectivas. Los hitos fundamentales del recorrido que he planteado son los siguientes. Rorty sortea 
los peligros que para él representan el escepticismo epistemológico y el esencialismo con una tesis antiesencialista panrelacionista. El análisis de este programa ontológico panrelacionista desemboca en un pluralismo descriptivo tanto a nivel intrarregional como transregional. Ambos tipos, especialmente el segundo, hacen difícilmente inteligible la tesis de la independencia causal del mundo que queda seriamente comprometida cuando intentamos hacerla funcionar en concreto determinando qué mundo es el que es causalmente independiente de nosotros. Parece que según describamos el mundo así será aquello que consideremos independiente de nosotros. Finalmente, he analizado una división ontológica fundamental que propone Rorty que, además de ser incompatible con el panrelacionismo y el pluralismo descriptivo, plantea en sí misma dificultades que la hacen inviable.

Óscar González-Castán

Universidad Complutense de Madrid

oscar.gonzalez@filos.ucm.es

\section{BIBLIOGRAFÍA}

AuxIER, R.E. y HAHN, L.E. (eds.) (2010): The Philosophy of Richard Rorty, The Library of Living Philosophers, XXXII, Chicago: Open Court.

Bilgrami, A. (2000): "Is Truth a Goal of Inquiry? Rorty and Davidson on Truth", en R.B. Brandom (ed.), Rorty and His Critics, Mass.: Malden, pp.242-262.

COLOMINA, J.J. y RAGA, V. (eds.) (2010): La filosofía de Richard Rorty. Entre pragmatismo y relativismo, Madrid: Biblioteca Nueva.

CometTI, J-P. (2010): "Rorty, Pluralistic Pragmatism, and Relativism”, en R.E. Auxier y L. E. Hahn (eds.), The Philosophy of Richard Rorty. The Library of Living Philosophers, Vol. XXXII, Chicago: Open Court, pp. 155-171.

DAvidson, D. (1990), "The Structure and Content of Truth", Journal of Philosophy, Vol. 87, pp. 279-328.

DAvidson, D. (2000): "Truth Rehabilitated", en R.B. Brandom (ed.), Rorty and His Critics, Mass.: Malden, pp. 65-74.

Del CASTILlo, R. y LÓPEZ, D. (2014): "Cuidarnos a/de nosotros mismos. El pensamiento de Richard Rorty (1931-2007)", en C. de Peretti y C. Rodríguez (eds.), 12 pensadores (y uno más) para el siglo XXI, Madrid: Dykinson, 2014, pp. 169-197.

FAernA, A.M. (2014): "Rorty and Dewey on Warrant", Contemporary Pragmatism, 11.1, pp. 15-26. 
GARCÍA-LORENTE, J.A. (2012): Richard Rorty: una alternativa a la metafísica occidental, Barcelona: Laertes.

GARCÍA-LORENTE, J.A. (2011): “El tratamiento neopragmatista de la verdad”, Endoxa, 27, (2011), pp. 295-315.

GEERTZ, C. (1973): The interpretation of Cultures, New York: Basic Books.

GONZÁlEZ-CASTÁN, O. L. (2014): “Consenso como sinécdoque y consenso como signo. Una crítica a la concepción rortyana de la verdad, la justificación y el consenso", Revista de Filosofía, Vol. 39, no 2 (2014), pp. 1-24.

Habermas, J. (2000): "Richard Rorty's Pragmatic Turn”, en R.B. Brandom (ed.), Rorty and His Critics, Mass.: Malden, 2000, pp. 31-55.

HusserL, E. (1954): Die Krisis der europäischen Wissenschaften und die transzendentale Phänomenologie. Hua VI., en W. Biemel (ed.), La Haya: Martinus Nijhoff.

JAmes, W. (1907/1975): Pragmatism and the Meaning of Truth, Cambridge, Mass.: Harvard University Press.

KALPOKAS, D. (2005): Richard Rorty y la superación pragmatista de la filosofía, Buenos Aires: Del Signo.

MACINTYRE, A. (1990): "Review of Contingency, Irony, and Solidarity by Richard Rorty", The Journal of Philosophy, Vol. 87, n. 12, pp. 708-711.

MCDowell, J. (2000): “Towards Rehabilitating Objectivity”, en R.B. Brandom (ed.), Rorty and His Critics, Mass.: Malden, pp. 109-122.

RAMBERG, B. (2014): "Davidson and Rorty: Triangulation and AntiFoundationalism", en J. Malpas y H.-Helmuth Gander (eds.), Routledge Companion to Hermeneutics, Londres: Routledge, pp. 216-235.

RAmberg, B. y FERGESTAD, I. (2011): “Triangulation: A Rationalizing Proposal", en M.C. Amotetti y G. Preyer (eds.), Triangulation. From an Epistemological Point of View, Frankfurt: Ontos Verlag, pp. 221-256.

RORTY, R. (1989): Contingency, Irony, and Solidarity, Cambridge: Cambridge University Press.

RORTY, R. (1991a): “Is natural science a natural kind?”, en R. Rorty, Objectivity, Relativism, and Truth. Philosophical Papers, vol.1, Cambridge: Cambridge University Press, pp. 46-62.

RORTY, R. (1991b): “Texts and Lumps”, en R. Rorty, Objectivity, Relativism, and Truth, Philosophical Papers, Vol.1, Cambridge: Cambridge University Press, Cambridge, pp. 78-92.

RORTY, R. (1991c): "Inquiry as Recontextualization: An Anti-dualist Account of Interpretation", en R. Rorty, Objectivity, Relativism, and Truth. Philosophical Papers, vol.1, Cambridge: Cambridge University Press, 1991c, pp. 93-110.

RorTy, R. (1991d): "Pragmatism, Davidson and Truth", en R. Rorty, Objectivity, Relativism, and Truth. Philosophical Papers, vol.1, Cambridge: Cambridge University Press, pp. 126-150. 
RORTY, R. (1993): "Putnam and the Relativist Menace", The Journal of Philosophy, Vol. XC, $n^{\circ}$ 9, pp. 443-461.

RORTY, R. (1995): "Is Truth a Goal of Inquiry? Davidson vs. Wright", The Pbilosophical Quaterly, Vol. 45, n 180 , pp. 281-300.

RORTY, R. (1998a): "Daniel Dennett on Intrinsicality", en R. Rorty, Truth and Progress, Philosophical Papers. Vol. 3, Cambridge: Cambridge University Press, pp. 98121.

RORTY, R. (1998b): "Feminism and Pragmatism", en R. Rorty, Truth and Progress, Philosophical Papers, Vol. 3, Cambridge: Cambridge University Press, pp. 202-227.

RorTy, R. (1999): "Ethics without Principles", en R. Rorty, Philosopby and Social Hope, Londres: Penguin, pp. 72-90.

RORTY, R. (2000): "Panrelacionismo", en R. Rorty El pragmatismo, una versión. Antiautoritarismo en epistemología y ética, Barcelona: Ariel, pp.139-167.

RORTY, R. (2000b): "Universality and Truth", en R.B. Brandom (ed.), Rorty and His Critics, Mass.: Malden, pp. 1-30.

RorTY, R. (2000c): "Response to Donald Davidson", en R.B. Brandom (ed.), Rorty and His Critics, Mass.: Malden, pp. 74-80.

RORTY, R. (2000d): "Response to McDowell", en R.B. Brandom (ed.), Rorty and His Critics, Mass.: Malden, pp. 123-128. 